\title{
Various surgical strategies for hepatocellular carcinoma located in caudate lobe
}

\author{
Jeong-Moo LEE, Kyung-Suk SUH*, Kwangpyo HONG, Eui Soo HAN, Sanggyun SUH, Su young HONG, \\ Suk Kyun HONG, YoungRok CHOI, Nam-Joon YI, Kwang-Woong LEE, Jae-Hyung CHO \\ Department of Surgery, Seoul National University Hospital, Seoul, Korea
}

Introduction: Caudate lobes are anatomically located between the hepatic hilum and inferior vena cava. Isolated caudate lobectomy is still a challenging procedure for hepatobiliary pancreas surgeons because it is quite complicated depending on the relationship between the surrounding major vascular structures and the biliary tract. In this study, we introduce a various surgical strategy for hepatocellular carcinoma located in the caudate lobe, and report the results.

Methods: From January 2005 to December 2015, 35 patients who underwent caudate lobectomy due to hepatocellular carcinoma in Seoul National University Hospital were enrolled. We described several surgical strategies with hanging maneuver and compared the clinical outcomes between the radical resection group and the local resection group.

Results: A total of 35 patients underwent hepatectomy including the caudate lobe. The median follow-up period was 86.7 months (3.8-183.6 months). There was 2 (5.7\%) extended right hemihepatectomy, 2 (5.7\%) extended left hemihepatectomy, 2 (5.7\%) right posterior sectionectomy including caudate lobe, 12 (34.3\%) cases of isolate caudate lobectomy, and 15 (42.9\%) cases of local tumorectomy (non-anatomical). There was no statistically significant differences were observed in operative time, hospital stay, and complication rate (mean, $247.1 \pm 104.7$ minutes vs. $247.1 \pm 104.7$ minutes, $p=0.729$; mean, $10.2 \pm 6.68$ days vs. $10.2 \pm 6.14$ days, $p=1.000$ ). The rates of recurrence were significantly lower in the radical resection groups than in the local resection group $(13 / 15,86.7 \%$ vs. $10 / 20,50.0 \% ; p$ $=0.034)$.

Conclusions: Various surgical strategies are necessary to resect caudate tumors depending on the location and degree of invasion. These several methods would be helpful to reduce recurrence without complications. 